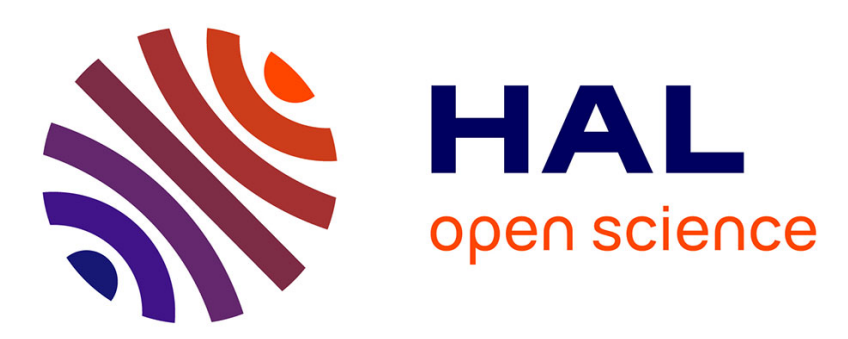

\title{
Status of the construction of the ATLAS electromagnetic liquid argon calorimeter, overview of beam test performance studies
}

\author{
L. Serin
}

\section{- To cite this version:}

L. Serin. Status of the construction of the ATLAS electromagnetic liquid argon calorimeter, overview of beam test performance studies. International Conference on Calorimetry in High Energy Physics CALOR2004 11, Mar 2004, Perugia, Italy. pp.210-217. in2p3-00023788

\section{HAL Id: in2p3-00023788 \\ https://hal.in2p3.fr/in2p3-00023788}

Submitted on 17 Feb 2005

HAL is a multi-disciplinary open access archive for the deposit and dissemination of scientific research documents, whether they are published or not. The documents may come from teaching and research institutions in France or abroad, or from public or private research centers.
L'archive ouverte pluridisciplinaire HAL, est destinée au dépôt et à la diffusion de documents scientifiques de niveau recherche, publiés ou non, émanant des établissements d'enseignement et de recherche français ou étrangers, des laboratoires publics ou privés. 


\title{
STATUS OF THE CONSTRUCTION OF THE ATLAS ELECTROMAGNETIC LIQUID ARGON CALORIMETER, OVERVIEW OF BEAM TEST PERFORMANCE STUDIES
}

\author{
LAURENT SERIN ${ }^{*}$ \\ Laboratoire de l'Accélérateur Linéaire, IN2P3-CNRS, Université de Paris-Sud, BP34, \\ 91898 Orsay Cedex, France
}

\begin{abstract}
The status of the mechanical construction of the ATLAS Liquid Argon electromagnetic calorimeter, near its completion, is presented with some emphasis on the quality control measurements which impact on the future performance of this detector. A selection of the calorimeter performance, obtained in beam test and demonstrating its ability to participate to the ATLAS particle identification taking advantage of its granularity or its precise time measurement, is shown. The response to muons particles, expected to provide a powerful tool during the commissioning phase, is also detailed.
\end{abstract}

\section{Introduction}

One of the main objectives of the LHC program is the search of the Higgs boson. The electromagnetic calorimeter has been primarily designed to meet this goal and thus to optimally detect Higgs boson decays in photon or electron/positron pairs. The calorimeter design requirements are the following:

- Good resolution of the energy measurements of photons and electrons: typically $10 \% / \sqrt{\mathrm{E}} \oplus 0.7 \%$. This requirement involves some constraints on the mechanical design and electronics calibration.

- Linearity of the response better than $0.1 \%$ which requires a good electronics calibration and a device to correct for upstream material energy loss.

- Particle identification ( $\mathrm{e}^{ \pm} / \mathrm{jets}$ or $\gamma / \pi^{0}$ ) and measurement of the direction of photons. These requirements lead to the design of a fine lateral and longitudinal segmentation.

- A large dynamic range (from $20 \mathrm{MeV}$ to $2 \mathrm{TeV}$ per channel) and an accurate time measurement which characterize the performance of the read out electronics.

To fulfill these criteria, and having to operate in a high radiation level environment, the ATLAS collaboration has chosen an already proven technique, a lead/liquid argon sampling calorimeter, with an innovative accordion geometry. After a short description of the calorimeter geometry and signal reconstruction in

\footnotetext{
* On behalf of the ATLAS Liquid Argon electromagnetic calorimeter group
} 
Section 2, selected quality control measurements during the production are described. In Section 3, the advantages of a longitudinal and a fine lateral granularity are illustrated with beam test results. The performance of time and muon signal measurements are also presented as they are key elements in the commissioning phase with cosmic muons.

\section{Calorimeter construction}

\subsection{Calorimeter description}

The calorimeter is a lead/liquid argon sampling calorimeter with accordion shaped electrodes and absorbers interleaved as sketched in Figure 1. Such geometry allows a very good hermeticity and a natural segmentation both lateral and longitudinal.

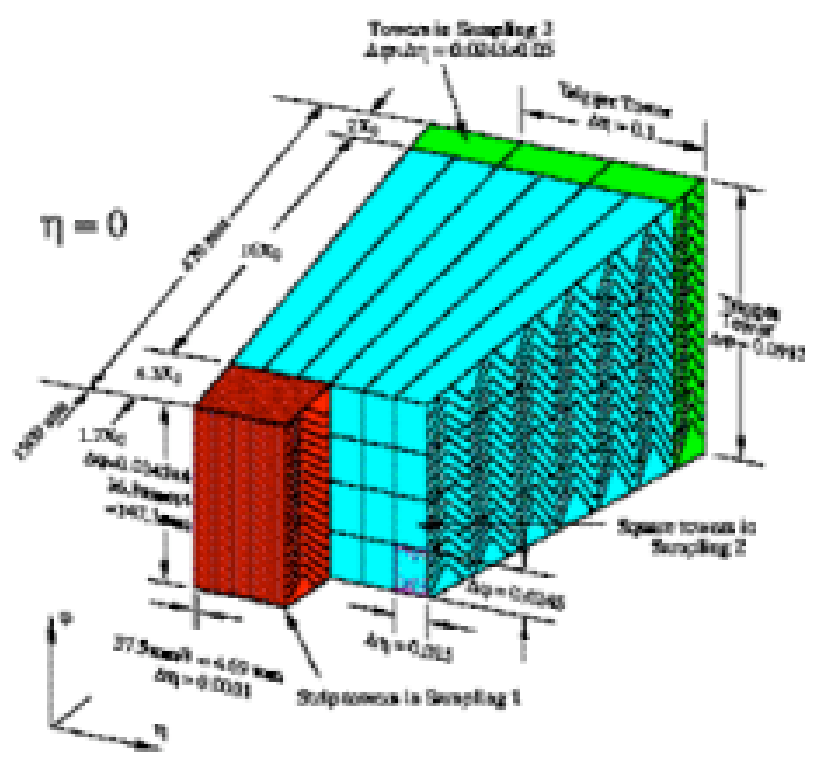

Figure 1: Sketch of the ATLAS electromagnetic barrel liquid argon calorimeter.

The barrel calorimeter is a $6.4 \mathrm{~m}$ long cylinder covering the rapidity range \pm 1.4 . It is composed of two half barrel containing 16 identical modules. Each module contains 64 electrodes and absorbers. The $\varphi$ granularity is obtained by grouping the electrode signal ( 4 in middle/back layer, 16 in strips) while the pseudo rapidity and depth granularity is obtained by etched patterns on the electrode itself. The first layer, optimized to end at about $5 \mathrm{X}_{0}$ including upstream 
material in ATLAS configuration, has a fine $\eta$ segmentation $(\sim 0.003$ equivalent to $\sim 5 \mathrm{~mm}$ at $\eta=0$ ) while rather coarse in the other direction $(0.1)$. The second layer (middle) contains most of the shower energy and ends after $\sim 22 \mathrm{X}_{0}$; its granularity is $\Delta \eta \times \Delta \varphi=0.025 \times 0.025$. Finally the back layer complements the calorimeter in radius and has twice granularity in $\eta$ of the middle layer.

The endcap calorimeter also uses the accordion shape geometry and has similar granularity up to $\eta=2.5$ in the outer wheel. The inner wheel $(\eta=2.5-3.2)$ has only two layers. In the barrel, the $2.1 \mathrm{~mm}$ argon gap size is supplied by a $2000 \mathrm{~V}$ voltage. In order to maintain a uniform resolution sampling term over the entire rapidity range, the lead thickness decreases from 1.5 to $1.1 \mathrm{~mm}$ at $\eta=0.8$. In the endcap, the lead thickness is constant in the outer wheel but the gap size increases with the radius. This effect is compensated to first order by the sampling fraction variation and operating with a constant electric field ensures almost a uniform response with respect to the radius.

Up to 1.8 in rapidity, a presampler with a coarse granularity is located before the calorimeter to compensate for the upstream material energy loss.

Further details about the mechanical description of these detectors can be found in [1].

\subsection{Signal collection and reconstruction}

When charged particles cross the gap, the ionization signal in the argon gives rise under electric field to a triangular current whose initial value $I_{0}$ is proportional to the incoming particle energy. This signal, collected in the inner layer of the electrodes via capacitive coupling, is sensitive to:

- The calorimeter sampling fraction and the lead/liquid argon gap thickness uniformity. Especially in the $\varphi$ direction the accordion geometry introduces a local variation which needs to be corrected for.

- The high voltage. In the barrel no effect is expected while in the endcap the needed continuous electrical field can not be achieved. The high voltage is thus constant by region of size typically 0.2 or 0.1 in $\Delta \eta$ and a residual slope response needs to be corrected in each region versus $\eta$.

- The argon temperature with sensitivity of $-2 \% /{ }^{\circ} \mathrm{c}(1 / 3$ from argon density and $2 / 3$ from drift velocity variations). A static gradient of about $1^{\circ} \mathrm{c}$ is expected in ATLAS cryostats. Probes, accurate at $10 \mathrm{mK}$, will be used to map the temperature.

- The attachment with impurities: a purity monitor in ATLAS will be used. The pollution level observed in the numerous cold and beam tests was at a few tenth of ppm.

- Due to the pile-up at high luminosity at LHC, ion build-up effects may appear. Dedicated studies have shown that such effects should remain negligible up $\eta=2.5$. 
The calorimeter signal is transmitted from the electrode connectors outside the cryostat via cables and feedthroughs. Front End boards located above the feedthroughs amplify, shape ${ }^{\dagger}$, store up to a level 1 accept and digitize the signal. As described in [2], the physics signal and the calibration signal (Figure 2) differs by the shape (triangular versus exponential) but also by the presence of an inductance on the electrode signal path which leads to an amplitude bias and a calibration shape distortion as a function of $\eta$. This effect amounts to about $0.15 \% / \mathrm{nH}$ and is taken into account in the signal reconstruction procedure which is based on digital filtering method [3].

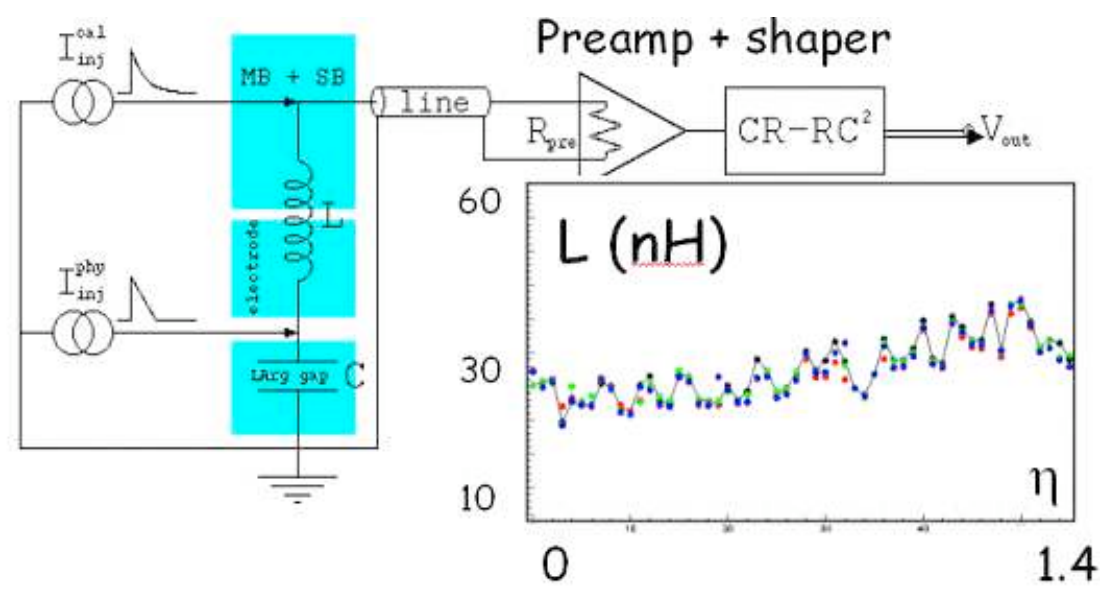

Figure 2: Electrical description of a calorimeter cell with calibration and ionization signal injection (left). Variation of the inductance seen by the middle layer cell versus $\eta$ in the barrel calorimeter (right).

\subsection{Quality control measurements}

To achieve the $0.7 \%$ overall constant term, many parameters were measured and monitored along the production. For instance Figure 3 shows the lead thickness variation, averaged over five absorbers, for the barrel calorimeter versus $\varphi$. The 3 $\%$ dispersion can be translated into a $1.8 \%$ contribution to the calorimeter constant term. Similarly from indirect gap variation measurements, a $1 \%$ contribution to the constant term is expected. Each of the 32 barrel and 16 endcap modules have been tested at cold over the entire construction period of 3 years. During the few days High Voltage (HV) tests, two types of problems were observed:

\footnotetext{
${ }^{\dagger}$ The shaper has a three gain structure in the ratio 1:10:100 to accommodate the 16 bits dynamic. The peaking time of a typical middle layer cell is $\sim 40 \mathrm{~ns}$ after shaping.
} 
- Short observed at cold and still present at warm. The faulty electrode in the sector ${ }^{\ddagger}$ can be identified and isolated on an individual HV line. It represents only $0.8 \%$ of all barrel electrode sectors and $1.5 \%$ of the endcap wheel. This is not an acceptance loss but a small local correction will need to be applied.

- When warming the module, the short disappears. In this case, the faulty electrode side sector is supplied by two different lines reducing by a factor two the potential area with no or not nominal HV.

Never simultaneous HV failures of both side of an electrode, which would result in a loss of acceptance, were observed. The absence of high voltage on one side in a sector can be recovered to first order by a factor two on the signal at the expense of a small increase of the noise and the $\varphi$ modulation amplitude [4].

During each cold test of individual modules and with the calorimeter fully assembled, all channels have been pulsed through the calibration line. For the barrel the total number of dead channels amounts to $0.14 \%$ of the almost $110 \mathrm{k}$ channels. In the first endcap wheel, this number is $0.5 \%$ still below the $1 \%$ specification.
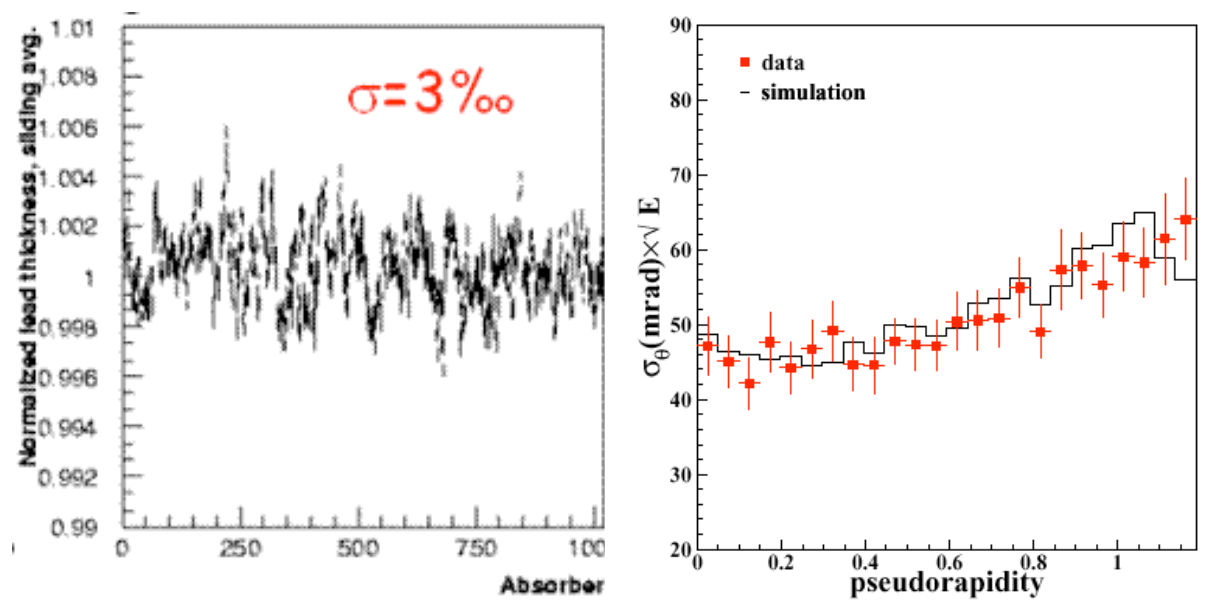

Figure 3: Normalized lead thickness averaged over five absorbers versus absorber number over the full $\varphi$ coverage (left), angular resolution over the barrel calorimeter coverage for $245 \mathrm{GeV}$ electrons.

\footnotetext{
* A barrel HV sector size is $\Delta \eta \mathrm{x} \Delta \varphi=0.2 \mathrm{x} 0.2$ i.e. 32 electrodes. Each electrode side is supplied by a different $\mathrm{HV}$ voltage line.
} 


\section{Beam test performance}

The exposure of the module 0 prototype in 1999 and 2000 to beam tests has been essential to finalize the calorimeter design: problems affecting mainly the physics signal, and not visible with electrical tests only, were detected. Four barrel and three endcap modules from the regular production have been exposed to beam particles to assess the calorimeter performance and validate the production. The main objective was the assessment of the response uniformity. The whole module acceptance was scanned with $245 \mathrm{GeV}$ (120 GeV) electrons for the barrel (endcap) modules. As detailed in [4], a $1 \%$ dispersion online could easily be reached while a $0.7 \%$ overall constant proved to be a rather demanding task. Dedicated studies have also been performed: a linearity study with a precise beam energy measurement [5], the $\gamma / \pi^{0}$ rejection with a dedicated photon beam, a dedicated setup for time measurement...

\subsection{First layer fine segmentation}

The fine segmentation of the first layer is designed to improve the measurement of the EM showers direction and the particle identification. This is especially relevant for the Higgs search when it decays into two photons. In order to minimize the contribution of the photon pair angle to the invariant mass resolution, the photon angle, i.e. the longitudinal LHC interaction point, has to be measured. Using electron beams, the particle position has been measured in the first layer section and the middle layer using an energy weighted barycenter ${ }^{\S}$. Combining these two measurements, the angular resolution has been extracted and is presented in Figure 3 as a function of the rapidity in the barrel reaching about $50 \mathrm{mrad} / \sqrt{ } \mathrm{E}^{* *}[6]$.

A large potential background to the $\mathrm{H} \rightarrow \gamma \gamma$ is due to jet-jet $(\gamma$-jet) events where a jet is misidentified as a photon, especially when the jet fragments into a leading $\pi^{0}$. Discriminating variables based on the shower shape in the first layer provide further rejection against these events. Using a dedicated setup to produce photons in the beam line, events faking a $50 \mathrm{GeV}$ transverse momentum $\pi^{0}$ have been emulated by superimposing offline photon shower shapes. The measured $\pi^{0}$

\footnotetext{
$\S$ For the middle layer a linear energy weighted barycenter is used and a S-shape correction is applied, while for the strip layer a logarithms energy weighted method is good enough.

${ }^{* *}$ With these numbers, the resolution expected in ATLAS on the longitudinal vertex position is $5 \mathrm{~mm}$ to be compared to the expected LHC dispersion of $56 \mathrm{~mm}$.
} 
rejection shown in Figure 4 is $(3.54 \pm 0.12)$ for a $90 \%$ efficiency to isolated photons, in good agreement with the MC expectation of $(3.66 \pm 0.12)^{\dagger \dagger}$
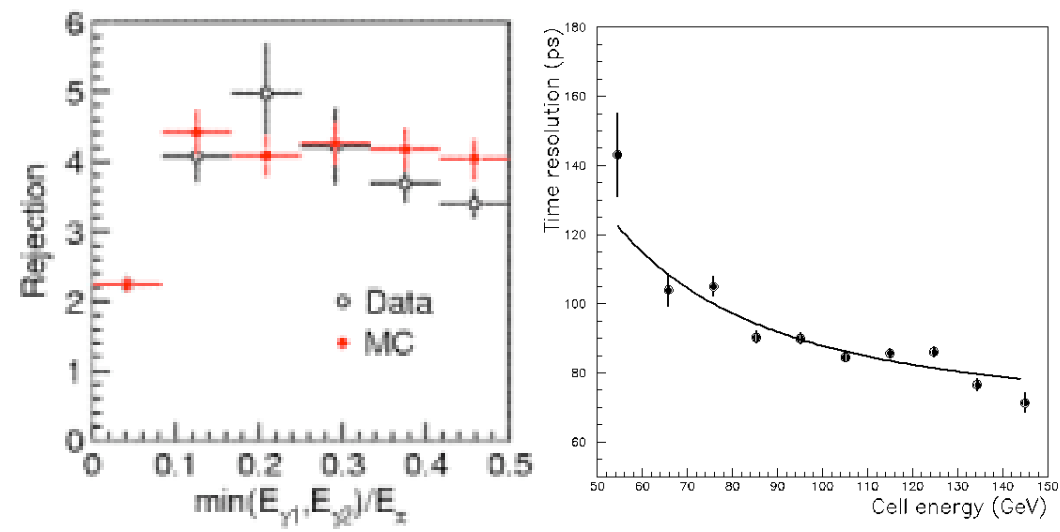

Figure 4: Rejection against $50 \mathrm{GeV}$ transverse momentum $\pi^{0}$ as a function of the fraction of energy taken by one photon (left), time resolution as a function of the cell energy for $245 \mathrm{GeV}$ electrons.

\subsection{Time measurement}

The ionization signal rise time in the argon is intrinsically fast and uniform, and should allow an accurate time measurement which can in turn be used to measure the decay time of long lived neutral particle (for instance in GSMB model)[7]. The excellent time measurement performance of the front end electronics with a constant term of about $20 \mathrm{ps}$, are not a limitation. The time resolution as a function of the cell energy has been measured with $245 \mathrm{GeV}$ electrons (Figure 4) and remains better than 100 ps for energies larger than $60 \mathrm{GeV}$, with a constant term of about $70 \mathrm{ps}$ [8]. This number is explained by the residual contributions of a few corrections needed to be applied.

\footnotetext{
${ }^{i \dagger}$ This number can not easily be extrapolated to the ATLAS setup due to the different amount of material in front of the calorimeter and to the presence of a magnetic field.
} 


\subsection{Muons and calorimeter commissioning}

The response to muons has been measured in beam tests and turned out to be a powerful too $^{\$}$. The signal over noise ratio is better than 7 across the barrel and throughout the endcap rapidity coverage in the middle layer. The time resolution is about $6 \mathrm{~ns}$ and a position measurement better than $5 \mathrm{~mm}$ is achieved in the $\varphi$ direction. Moreover using a search algorithm for the first layer strip hit by the muon with $\mathrm{S} / \mathrm{B} \sim 5$, an $\eta$ resolution of $5 \mathrm{~mm}$ can also be obtained.

In 2006, a first run with cosmic rays should take place with a typical rate $\sim 1 \mathrm{~Hz}$ for projective muons during a few months. The performance reached for muons in beam tests should allow to align the physics pulse to $1 \mathrm{~ns}$ accuracy and to check the signal reconstruction uniformity (LC effect) at the $0.3 \%$ level.

\section{Conclusion}

Following almost 10 years of R\&D, the ATLAS Liquid Argon calorimeter construction and assembling, which started in 2001, are now completed. The electrical and HV tests have shown very little problem. The calorimeters now inserted in the cryostat will be tested at cold in surface in 2004 before being transferred in the pit. A five year program of beam tests has been brought to its end and was essential both to finalize the calorimeter design with prototypes and to validate the module production. Numerous results leading to an important gain of experience were obtained from these tests. This knowledge will be further used during the commissioning phase in order to have a fully operational calorimeter at the start of the LHC.

\section{References}

1. ATLAS Liquid Argon Technical Design Report, CERN/LHCC/96-40 ATLAS Liquid Argon Technical Design Report, CERN/LHCC/96-41

B. Aubert et al., Nucl. Instr. and Meth. A500 (2003) 178-201

B. Aubert et al., Nucl. Instr. and Meth. A500 (2003) 202-231.

2. C. de La Taille, contribution to these proceedings

3. W.E Cleland, E. Stern, Nucl. Instr. Meth. A338 (1994) 467

4. O. Gaumer, contribution to these proceedings

5. G. Graziani, contribution to these proceedings

6. R. Sacco, ATL-LARG-2003-008

7. ATLAS detector and physics performance TDR, CERN/LHCC/99-15

8. I. Nikolic-Audit, L. Serin, ATL-LARG-2004-002

\footnotetext{
\# A problem of missing ground on the electrode of the first prototype was clearly detected only with a muon scan.
} 